\title{
Mapeamento e Identificação do Comportamento Sonoro de um Cajón Acústico
}

\section{Mapping and Identification of the sound behavior of an Acoustic Cajón}

Rafael Nardon Ferraresi ${ }^{1}$, Vinícius Abrão da Silva Marques², Paulo Balduino Flabes $\mathrm{Neto}^{3}$, Ricardo Humberto de Oliveira Filho ${ }^{4}$

\section{RESUMO}

Para realizar o mapeamento e avaliar o comportamento sonoro do cajón acústico, é necessário conhecer 0 instrumento, considerando o seu formato, materiais que 0 compõem e principalmente a maneira de manuseá-lo, considerando as variações que o músico pode realizar na forma de tocar. Neste trabalho realizou-se um teste de simetria sonora, verificando se um lado do tampo do instrumento (direito) emitiria 0 mesmo som que o seu lado oposto (esquerdo). Foi avaliada a influência da rigidez do tampo, mostrando que existe variações no som emitido quando se aplica alguma restrição em seu movimento. Verificou-se a existência de sobreposição quando o músico toca com as duas mãos no tampo no mesmo intervalo de tempo, fazendo com que o som emitido seja uma soma das regiões excitadas, e quando o músico toca 0 instrumento de maneira usual, foi verificada uma pequena sobreposição, classificada como irrelevante para a situação do instrumento tocado por longos intervalos de tempo. Os testes foram realizados com o auxílio de um pêndulo, que garantiu a repetitividade e o padrão de excitação, comprovada nos sinais adquiridos. Com isto conseguiu-se coletar dados suficientes para mapear e identificar acusticamente 0 instrumento, possibilitando o posterior projeto e fabricação de um cajón eletrônico.

Palavras-chave: Cajón acústico, Acústica, Mapeamento Sonoro

\section{ABSTRACT}

In order to perform the mapping and to evaluate the sound behavior of the acoustic cajón, it is necessary to know the instrument, considering its format, the materials that compose it and mainly the way to handle it, considering the variations that the musician can perform when play it. In this work, a sound symmetry test was performed, verifying if one side of the instrument (right) emitted the same sound from the opposite (left) side. The influence of the stiffness of the top was evaluated, showing that there are variations in the sound emitted when some restriction is applied in its movement. It confirms there is overlap when the musician plays with both hands at the top in the same time, making the sound emitted a sum of the excited regions. When the musician touches the instrument in the usual way there is a small overlap, classified as irrelevant to the situation of the instrument touched by long intervals of time. The tests were performed with the aid of a pendulum, which ensured repeatability and excitation pattern, proven in the acquired signals. With this, it was possible to collect enough data to map and identify the instrument acoustically, enabling the design and subsequent manufacture of an electronic cajón.

Keywords: Acoustic cajón, Acoustic, Sound mapping

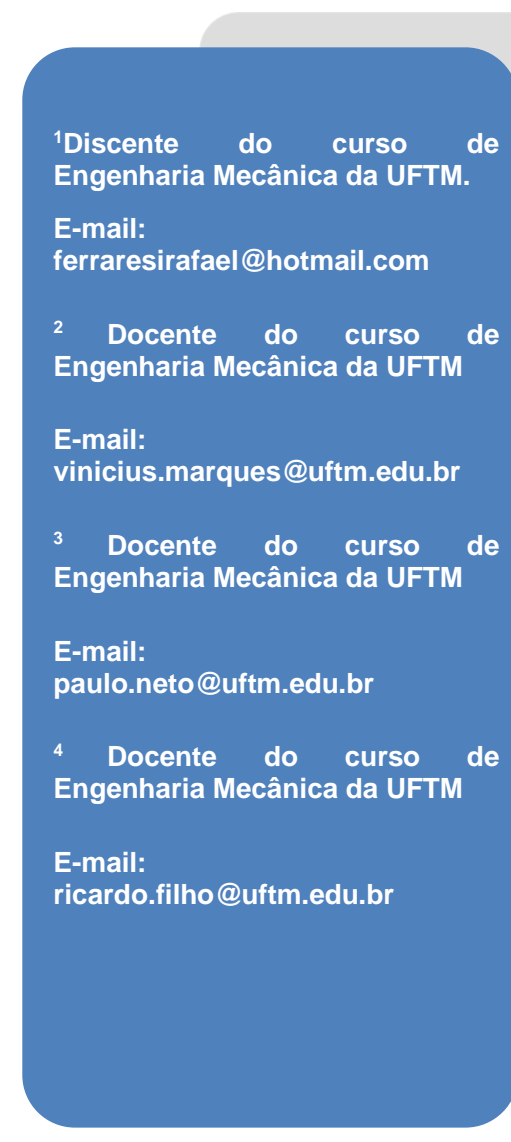




\section{INTRODUÇAOO}

Os instrumentos eletrônicos por décadas vêm evoluindo e disseminando-se no meio artístico, podendo citar desde a criação do Tellarmonium, onde era necessário a utilização de rotores, ao EWI (Eletronic Wind Instrument), um instrumento puramente eletrônico que possibilita inúmeras variações sonoras. Esta evolução ocorre em todos os seguimentos de instrumentos, podendo ser um teclado, uma bateria eletrônica, um sampler, dentre vários outros, buscando inovar ou até mesmo recriando um instrumento convencional.

Neste contexto surgiu a ideia de construir um instrumento eletrônico que simulasse a sonoridade e a forma de tocar de um Cajón, um instrumento musical de estrutura simples, porém com muitas propriedades sonoras e que vem cada vez mais sendo utilizados por músicos em grandes apresentações. Para tanto, foi levantada a necessidade de realizar o mapeamento sonoro do instrumento, objetivando identificar as frequências emitidas por determinadas partes de um cajón acústico.

Segundo Santos (2006), um instrumento elétrico é aquele que utiliza de partes mecânicas para gerar o som e após ser amplificado é que se consegue ouvi-lo. Já um instrumento eletrônico é aquele em que o som é gerado por circuitos elétricos, sem a necessidade de partes mecânicas.

Segundo Cristino (2008), para tocar o cajón o instrumentista senta-se sobre o mesmo e golpeia o tampo laminado. Basicamente o instrumento possui dois tipos de sons, um mais grave, semelhante ao som do bumbo da bateria e um som mais agudo que se assemelha ao som da caixa da bateria, sendo que, para conseguir o som grave o toque deve ser dado no centro do tampo com a mão inteira em forma de concha e não se deve deixar a mão encostada no instrumento, e para conseguir o som agudo o toque deve ser dado em forma de "tapa" na borda superior do tampo e utiliza-se apenas as falanges dos dedos.

Além da maneira de tocar, existem outros fatores que interferem na qualidade do instrumento e consequentemente na qualidade do som emitido, como as suas dimensões e o tipo de madeira que o cajón é construído. Cavalcante (2006) em seu trabalho de pesquisa Avaliação de Novas Espécies Madeireiras na Fabricação de Cajón, para a Universidade de Brasília, realizou alguns testes acústicos em cajóns com diferentes tipos de madeiras, sendo elas o Marupá, o Freijó, o Ipê e utilizou de um cajón de compensado encontrado no mercado para comparar os resultados de decaimento de intensidade de cada cajón. Foram realizados testes acústicos em um estúdio profissional. Para os testes 
foi utilizado um pêndulo para que se pudesse excitar o tampo do cajón sempre com a mesma força e garantir também a mesma posição de excitação no cajón. Nos testes realizados no estúdio foram obtidos as respostas de amplitude no tempo e, com a análise de FFT (Fast Fourier Transform), observou-se o espectro de frequências. Com isto pôdese visualizar o decaimento de cada cajón e encontrar a melhor madeira para a produção de cajóns. Segundo o autor, o Freijó apresenta os melhores resultados, sendo altamente indicado para a fabricação de cajón.

Neste trabalho são apresentadas diferentes avaliações sonoras de um cajón. A primeira delas se baseia em uma análise de simetria sonora do instrumento. Em seguida foi verificada a influência do tampo ser excitado com algum tipo de bloqueio, sendo que bloquear o tampo em alguma região com uma das mãos ou com os pés é uma maneira de se tocar o instrumento. Por fim, verificou-se a existência ou não de sobreposição sonora quando o músico toca mais de uma região ao mesmo tempo ou com batidas alternadas.

Com todas as análises concluídas consegue-se, a partir deste trabalho, um embasamento para o projeto do cajón eletrônico, visto que o mesmo tem que ser o mais fiel possível, tanto na sonoridade como na maneira de tocar, em relação ao cajón acústico.

O objetivo deste trabalho portanto é mapear e identificar o espectro de frequências emitido pelo cajón a partir da excitação de seu tampo e, desta forma, obter um banco de dados sonoro para futuramente desenvolver um instrumento digital na forma semelhante ao instrumento acústico.

\section{MATERIAIS E METODOS}

Para se realizar o mapeamento das frequências emitidas pelo cajón, foram avaliados inicialmente três fatores:

1- A forma de excitação do cajón de maneira a garantir a repetibilidade dos testes;

2- A simetria do seu formato de caixa;

3- As diferentes maneiras que o instrumento pode ser tocado, podendo ser:

3.1) Excitação com restrição em parte do tampo;

3.2) Excitação de duas regiões simultaneamente;

3.3) Excitação de duas regiões sequencialmente. 
Para avaliar os sinais adquiridos, foram calculadas as suas FFT's (Transformada Rápida de Fourier). Os espectros de frequência resultantes foram então comparados de acordo com o Coeficiente de Correlação.

O Coeficiente de Correlação é uma medida que mostra o grau de relação entre duas ou mais variáveis. A correlação pode ser linear simples, quando a relação entre duas variáveis $\mathrm{X}$ e $\mathrm{Y}$ é proporcional, ou pode haver um correlação não-linear. Neste trabalho, a análise de correlação irá fornecer um número que indica como as variáveis variam conjuntamente, sem ao tanto investigar as causas e os efeitos destas variações (LIRA, 2004).

A seguir são descritos a instrumentação utilizada nos testes bem como as análises realizadas previamente para identificar a influência dos 3 fatores citados anteriormente. Em seguida são apresentados os resultados obtidos.

\subsection{INSTRUMENTAÇÃO UTILIZADA}

Todo o processo de aquisição e tratamento de dados foram realizados com a ajuda de programas computacionais desenvolvidos no MATLAB®, como a aquisição dos dados, a geração do espectro de frequência e a análise do coeficiente de correlação.

A aquisição de dados é um processo que consegue quantificar fenômenos elétricos e variáveis físicas com o uso de um computador, sensores e um hardware de digitalização dos sinais (NATIONAL INSTRUMENTS, 2016).

Para o software deve-se definir alguns parâmetros como a frequência de amostragem ( $\mathrm{Fs}$ ) que é a quantidade de vezes que se realiza a amostragem em uma unidade de tempo, o intervalo de amostragem (Ts) que é a duração total da coleta dos dados, o número de pontos ( $n$ ) que é a quantidade de amostras coleadas, o intervalo de tempo entre as amostras adquiridas (dt) e a resolução em frequência (df) que é fornece o espaçamento entre os dados no espectro de frequências.

A geração do espectro de frequências é obtida pela transformação do sinal adquirido no domínio do tempo, para um sinal no domínio da frequência, para isto é realizado a Transformada Rápida de Fourier - FFT do sinal adquirido.

O cajón utilizado nos testes é da marca FSA cajóns ${ }^{\circledR}$, Strike Series "The Sixties" SK 4000. O instrumento é produzido com compensado de Sumaúma, possui altura de 47 $\mathrm{cm}$, pesa $6,2 \mathrm{Kg}$ e possui uma esteira interna justaposta ao tampo de 12 fios (FSA CAJÓNS, 2016). 
Para realizar a aquisição dos dados utilizou-se uma placa de aquisição da marca National Instruments ${ }^{\text {inc }}$ CDAQ 9171, um microfone pré-amplificado da marca PCB Piezotronicsinc modelo 378B02, um computador, cabos e acessórios de medição.

O microfone foi mantido a $80 \mathrm{~cm}$ de distância em relação ao cajón, e no momento da aquisição o operador estava sentado sobre o instrumento, considerando que o mesmo fizesse parte do sistema.

O microfone foi ligado à placa de aquisição por um cabo coaxial de baixo nível de ruído com conexões $B N C / B N C$, e a placa foi ligada ao computador via USB. No computador, foi utilizado um programa para aquisição de dados com os seguintes parâmetros:

- Frequência de Amostragem: $25600 \mathrm{~Hz}$;

- Tempo de Aquisição: 10 segundos;

- Número de Pontos: 256000;

- Intervalo de amostragem: $3,906 \times 10^{-5}$ segundos;

- Resolução em frequência: $0,1 \mathrm{~Hz}$.

Para as análises, foram feitas 15 repetições de cada ensaio e, desenvolvido um outro programa para o pós processamento, calcular a FFT de cada sinal e calcular a média das FFT's.

\subsection{FORMA DE EXCITAÇÃO}

Durante os testes de excitação do tampo do cajón deve-se garantir a repetibilidade dos testes. Para tanto, foi necessário a construção de um pêndulo, que excita o tampo do cajón sempre com a mesma intensidade, tentando garantir que seja o mais próximo possível das características da mão humana e mantendo o mesmo local de impacto.

Foram testados quatro blocos de madeira de dois tamanhos diferentes na extremidade do pêndulo, sendo dois com revestimento em EVA. Os blocos grandes possuem a superfície de contato com dimensões de $8 \mathrm{~cm} \times 11 \mathrm{~cm}$ e os blocos pequenos $8 \mathrm{~cm} \times 7 \mathrm{~cm}$. Estes blocos foram acoplados ao pêndulo, havendo a possibilidade de regular o local em que o bloco irá golpear o tampo. Assim, pôde-se avaliar se o tamanho e o revestimento alterariam a sonoridade do instrumento. 
Para tanto, selecionou-se uma parte específica do tampo do cajón onde todos os blocos, acoplados ao pêndulo, realizaram a sua excitação. A Figura 1 traz os espectros de frequência gerados para cada teste realizado.

Após calculadas as transformadas de Fourier e obtidos os espectros de frequências, foi analisado o coeficiente de correlação considerando uma faixa de frequências de 0 a $250 \mathrm{~Hz}$, pois como pode-se ver na Figura 1, acima desta frequência a amplitude do som adquirido decai mais de $20 \mathrm{~dB}$. Os resultados para o coeficiente de correlação são mostrados na Tabela 1.

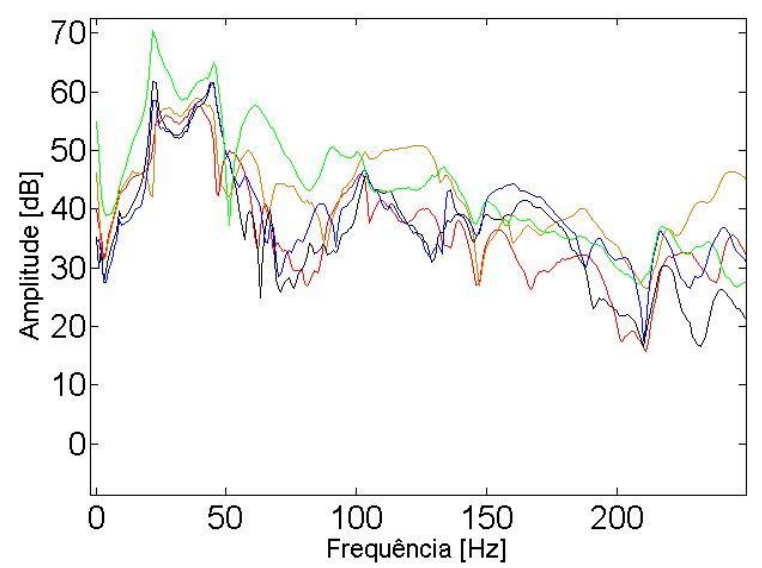

Figura 1. Espectros de frequências para os ensaios realizados para os quatro tipos de bloco e para a mão. (verde: mão; vermelho: bloco grande com EVA; amarelo: bloco grande sem EVA; magenta: bloco pequeno com EVA; azul: bloco pequeno sem EVA).

Tabela 1. Tabela dos Coeficientes de Correlação de cada bloco em relação à mão, em porcentagem [\%].

\begin{tabular}{cccc}
\hline \multicolumn{2}{c}{ Bloco Grande } & \multicolumn{2}{c}{ Bloco Pequeno } \\
Revestido & Não revestido & Revestido & Não revestido \\
\hline 82,27 & 69,93 & 78,28 & 74,51 \\
\hline
\end{tabular}

A partir dos dados apresentados na Tabela 1, pode-se constatar que o som emitido pelo instrumento quando tocado com os blocos revestidos de EVA assemelha-se mais ao som emitido pela mão.

Desta forma chegou-se à conclusão de que o bloco grande revestido em EVA se assemelha mais ao comportamento da mão humana, sendo adotado para os testes seguintes. 


\subsection{ANÁLISE DE SIMETRIA}

O ensaio de simetria verifica se ambos os lados do cajón emitem o mesmo tipo de som ao serem excitados.

No cajón foi demarcada uma linha central sobre o seu tampo dividindo-o em dois lados (esquerdo e direito), após isto, o instrumento foi excitado pelo bloco grande revestido em EVA em três regiões diferentes de cada lado, e assim pode-se comparar o espectro de frequências de cada região e averiguar se há simetria sonora no instrumento.

Foram definidas as regiões 1, 2 e 3 do lado direto e as regiões 4, 5 e 6 do lado esquerdo (referência olhando o cajón de frente), conforme Figura 2. A região 1 foi comparada com a região 4 estando ambas a $46 \mathrm{~cm}$ de altura com referência na base. $A$ região 2 foi comparada com a região 5 e ambas estão a $42 \mathrm{~cm}$ de altura em relação à base. A região 3 foi comparada com a região 6 , sendo que ambas estão a $30 \mathrm{~cm}$ de altura em relação à base.

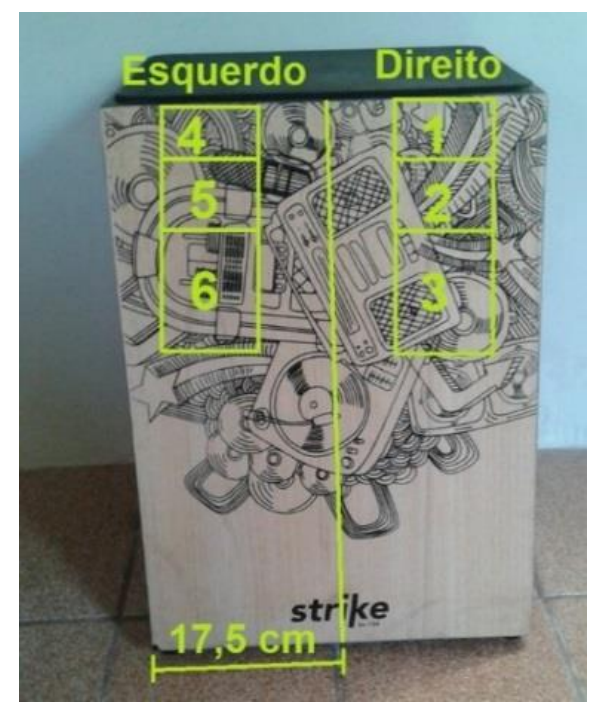

Figura 2. Posições onde o cajón foi tocado para o teste de simetria.

A Figura 3 mostra os espectros de frequência para as regiões 2 e 5 , na qual observa-se que ambos são relativamente semelhantes. 


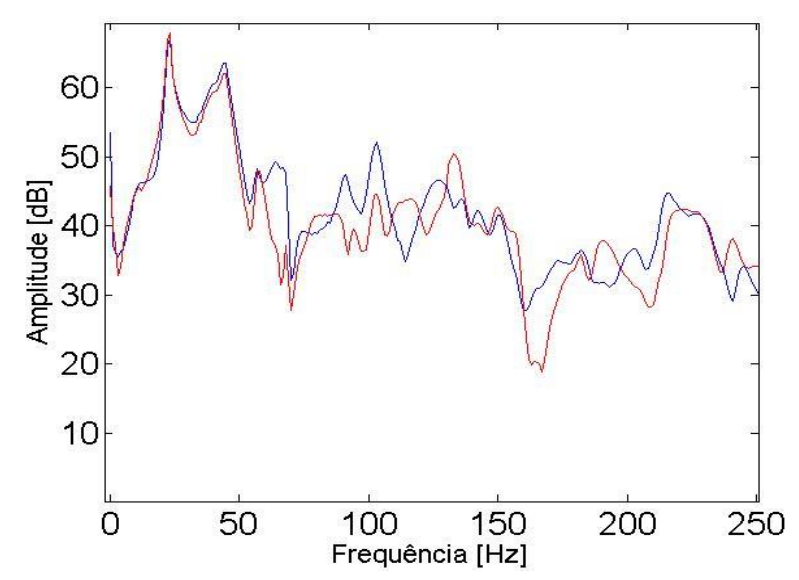

Figura 3. Espectro de frequências para as regiões 2 (azul) e 5 (vermelho).

Após calculados todos os espectros, fez-se a análise do coeficiente de correlação para a faixa de 0 a $250 \mathrm{~Hz}$. Os resultados são mostrados na Tabela 2.

Tabela 2. Coeficiente de correlação entre as regiões excitadas, em porcentagem [\%].

\begin{tabular}{ccc}
\hline Regiões 1 e 4 & Regiões 2 e 5 & Regiões 3 e 6 \\
\hline 82,3 & 86,1 & 83,5
\end{tabular}

É possível constatar que as regiões comparadas não apresentam diferenças significativas no som gerado. As diferenças observadas podem ser justificadas devido ao desgaste do instrumento e por ser um instrumento mais básico.

Sendo assim, pelos resultados das análises realizadas, pôde-se concluir que o instrumento é simétrico.

\subsection{EXCITAÇÃO COM RESTRIÇÃO EM PARTE DO TAMPO}

Para mapear o cajón, considerou-se a maneira que o instrumentista toca. Umas destas maneiras mais usual é apoiar uma das mãos sobre uma região do cajón e tocar em outra região, modificando a rigidez do tampo e gerando um som diferente do emitido quando o cajón é tocado livremente.

Desta forma, foram comparados os espectros de frequência para os sons gerados pelo instrumento quando se bloqueia uma região com os gerados quando o cajón é tocado livremente. Afim de se diferenciar tais regiões, criou-se uma malha $(3 \times 3)$ no tampo do cajón nas regiões mais tocadas pelo instrumentista. 
A Figura 4 representa a malha feita sob o tampo, localizada no topo do tampo e centralizada, com $31 \mathrm{~cm}$ de largura e $27 \mathrm{~cm}$ de altura. Todas as regiões demarcadas possuem o mesmo tamanho.

\begin{tabular}{|c|c|c|}
\hline 1 & 2 & 3 \\
\hline 4 & 5 & 6 \\
\hline 7 & 8 & 9 \\
\hline
\end{tabular}

Figura 4. Malha de regiões a serem excitadas.

Ao analisar um instrumentista tocando o cajón, verifica-se que o mesmo bloqueia somente as regiões $1,7,3$ ou 9 . Como o instrumento foi classificado como simétrico, considerou-se para os testes o bloqueio das regiões 3 e 9 , e com isto excitou-se as demais regiões.

Considerou-se ainda para a análise que, quando se bloqueia a região 3 com a mão esquerda, o instrumentista fica impossibilitado de tocar as regiões 6 e 9, portanto não houve a necessidade de realizar a aquisição do espectro para estas regiões com o bloqueio em 3. Já o bloqueio em 9 (realizado normalmente com o pé) possibilita ao instrumentista tocar todas as demais regiões, por ficar com as duas mãos livres. Sendo assim, para a análise, foram consideradas as combinações descritas a seguir:

- Bloqueio na região 3: Excitou-se as regiões 1, 2, 4, 5, 7 e 8;

- Bloqueio na região 9: Excitou-se as regiões 1 a 8;

- Tocado livremente: Excitou-se as regiões 1 a 9.

Após adquirir os sinais do som emitido pelo cajón ao bloquear as regiões descritas, foram gerados os espectros de frequência e comparados com os das regiões excitadas livremente. Lembrando que foram realizadas 15 repetições para cada região excitada e que o espectro apresentado é o médio. A Figura 5 exemplifica a comparação entre os espectros de frequências da região 1 tocada livremente e com bloqueio da região 3 . 


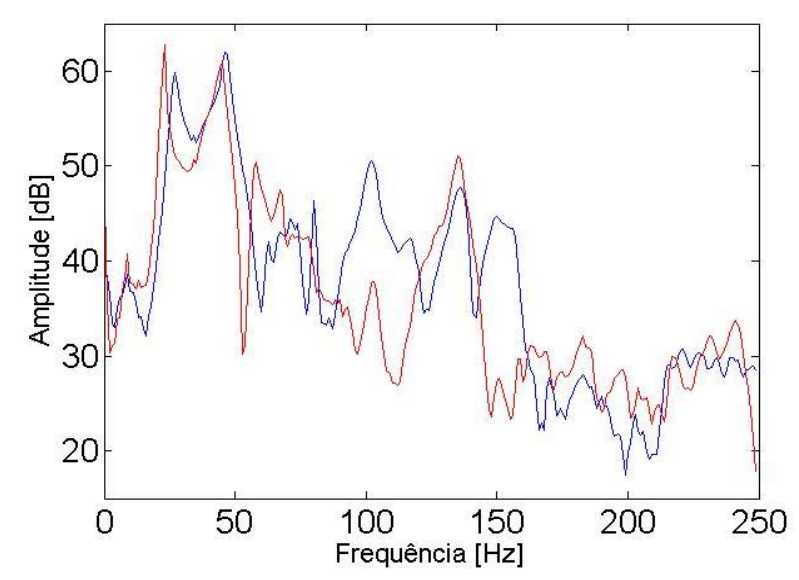

Figura 5. Espectros de frequência para a região 1 com bloqueio em 3 (azul) e tocado livremente (vermelho).

Foram realizadas as comparações de todas as regiões citadas anteriormente e em seguida realizadas as análises dos coeficientes de correlação entre as regiões comparadas. As Tabelas 3 e 4 representam as comparações com bloqueio nas regiões 3 e 9 respectivamente.

Tabela 3. Coeficiente de correlação entre os espectros do cajón tocado livremente e com bloqueio da região 3 , em porcentagem [\%].

\begin{tabular}{cccccc}
\hline \multicolumn{7}{c}{ Região tocada com bloqueio na região 3 } \\
\hline 1 & 2 & 4 & 5 & 7 & 8 \\
\hline 73,6 & 75,5 & 54,2 & 78,4 & 62,7 & 85,4 \\
\hline
\end{tabular}

Tabela 4. Coeficiente de correlação entre os espectros do cajón tocando-o livre e tocando-o com bloqueio na região 9 , em porcentagem [\%].

\begin{tabular}{cccccccc}
\hline \multicolumn{8}{c}{ Região tocada com bloqueio na região 9} \\
1 & 2 & 3 & 4 & 5 & 6 & 7 & 8 \\
\hline 82,2 & 80,2 & 75,8 & 80,8 & 74,5 & 78,5 & 68,2 & 70,9 \\
\hline
\end{tabular}

De acordo com as Tabelas 3 e 4 verifica-se que o coeficiente de correlação é baixo comparados os sons emitidos quando se toca livremente e o som emitido com o instrumento bloqueado em qualquer uma das regiões.

Pode-se então concluir que o som emitido pelo cajón com bloqueio não é o mesmo som emitido quando se toca livremente. 


\subsection{EXCITAÇÃO DE DUAS REGIÕES SIMULTANEAMENTE}

Após analisar a maneira que se utiliza o instrumento, percebeu-se que o mesmo pode ser tocado batendo as duas mãos juntas no tampo do cajón, o músico realiza este ato quando necessita de uma maior intensidade no som, sendo assim, se fez necessário avaliar se o sinal gerado é uma sobreposição de sons ou se o som de alguma região excitada torna-se predominante no sinal.

Para isto realizou-se os testes utilizando o mesmo mapa da análise anterior, como mostra a Figura 4, excitando as mesmas regiões, porém duas a duas. A seguir encontrase os pares de regiões excitadas simultaneamente, e a nomenclatura utilizada para identificar tais testes:

- Regiões 1 e $2:$ R1_2

- Regiões 1 e 5 : R1_5

- Regiões 1 e 3 : R1_3

- Regiões 1 e 6 :R1_6

- Regiões 4 e $2:$ R4_2

- Regiões 4 e 5 : R4_5

- Regiões 4 e 3 : R4_3

- Regiões 4 e 6 : R4_6

Como o instrumento foi considerado simétrico, não foi necessário excitar todos os pares, portanto se houver sobreposição sonora nestas combinações, seram consideradas que as combinações simétricas também sofrerão sobreposição.

Para cada par excitado, realizou-se 15 repetições, após adquirir todos os sinais foram calculadas as transformadas de Fourier e gerados os espectros de frequências com a média das 15 repetições de cada par excitado. Em seguida, afim de se verificar a existencia de sobreposição, foi realizada a soma dos espectros dos mesmos pares excitados quando tocado o instrumento livremente e assim, comparando os espectros gerados neste teste com os espectros obtidos ao tocar as regiões simultaneamente.

A Figura 6 mostra a comparação entre a soma dos espectros das regiões 4 e 6 tocadas livremente e o espectro obtido com as regiões 4 e 6 tocadas juntas. 
Em seguida foram calculados os coeficientes de correlação para todos os testes, apresentados na Tabela 5.

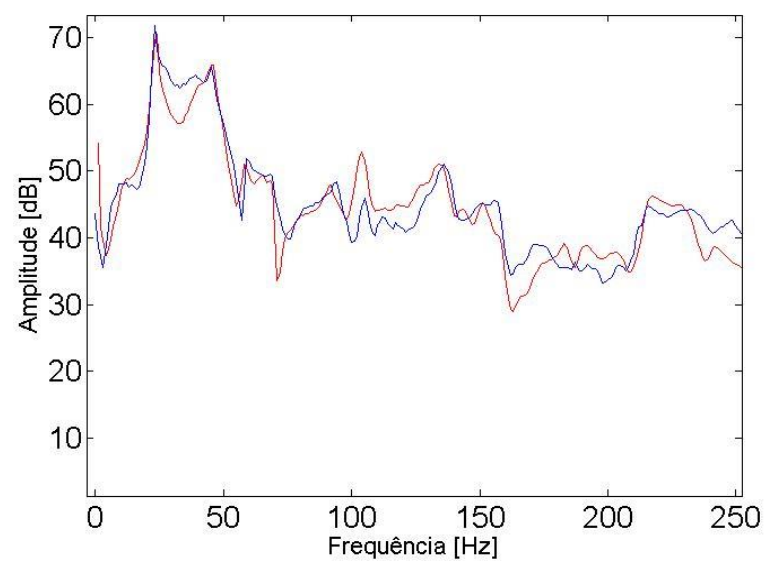

Figura 6. Espectros de frequências das regiões 4 e 6 somados (vermelho) e adquirido com as regiões tocadas juntas (azul).

Tabela 5. Coeficiente de correlação entre a soma dos espectros das regiões e os respectivos pares excitados juntos em porcentagem [\%].

\begin{tabular}{cccccccc}
\hline & \multicolumn{8}{c}{ Regiões excitadas simultaneamente } \\
1 e 2 & 1 e 3 & 1 e 5 & 1 e 6 & 4 e 2 & 4 e 3 & 4 e 5 & 4 e 6 \\
\hline 88,6 & 87,9 & 80,2 & 81,0 & 86,8 & 88,1 & 85,0 & 89,8 \\
\hline
\end{tabular}

A partir dos dados da Tabela 5, ao excitar duas regiões do tampo simultaneamente, percebe-se que o som emitido possui maior intensidade, porém não se consegue perceber a existência de alguma região predominante. Além disso, não se consegue distinguir as duas regiões excitadas com clareza, porém percebe-se que há mais de uma região sendo excitada.

Sendo assim, com base na análise dos resultados, pode-se dizer que o som gerado se assemelha a uma soma das regiões excitadas, sendo o sinal adquirido semelhante a uma sobreposição dos sons.

\subsection{EXCITAÇÃO DE DUAS REGIÕES SEQUENCIALMENTE}

A maneira mais usual de se tocar o instrumento é com as batidas alternadas (repique), uma mão na sequência da outra, em várias regiões do instrumento. Ao analisar 
um músico tocando constata-se que, ao se excitar o tampo com uma das mãos, logo na sequência, ainda com o tampo vibrando, pode-se ainda excitá-lo com a outra mão. Tal fato levantou a possibilidade da existência de sobreposição de sons neste estilo de tocar, pois como as batidas são realizadas com o tampo vibrando, ocorre uma alteração no sistema do cajón. Essa alteração que será analisada nesta seção.

Para realizar os testes foi utilizado o mesmo mapa sobre o tampo do cajón dos testes anteriores, mostrado na Figura 4.

O teste consiste em excitar o tampo em uma região e na sequência excitar o tampo em outra região, gerando assim um sinal no tempo com dois impulsos. Para a comparação, será feita a soma dos espectros de frequência destas mesmas regiões, porém excitadas individualmente, e analisadas a possibilidade de sobreposição.

As regiões excitadas foram 4 e 2, 4 e 5, e 4 e 6 , as demais regiões não foram excitadas devido ao fato do instrumento ser simétrico e considerando que se houver sobreposição ou não nestas regiões, as demais sofrerão os mesmos efeitos.

A Figura 7 mostra a soma dos espectros de frequência para as regiões 4 e 6 excitadas individualmente e excitadas sequencialmente.

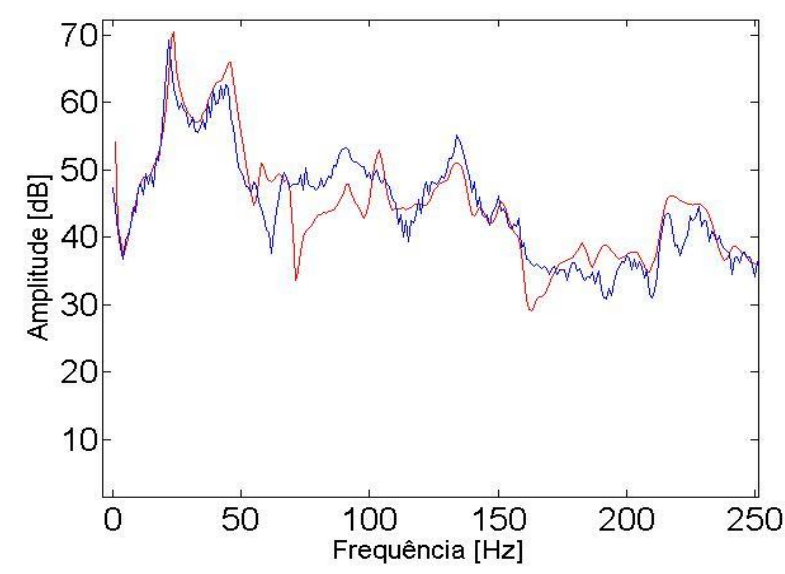

Figura 7. Soma dos espectros de frequência das regiões 4 e 6 excitadas individualmente (vermelho) e espectro de frequência das regiões 4 e 6 excitadas sequencialmente (azul).

A Tabela 6 mostra os coeficientes de correlação entre a soma dos espectros de frequências sobrepostos com o das regiões tocadas sequencialmente. 
Tabela 6. Coeficientes de Correlação entre a soma dos espectros das regiões e os respectivos pares excitados alternadamente, em porcentagem [\%].

\begin{tabular}{|c|c|c|}
\hline \multicolumn{3}{|c|}{ Regiões excitadas sequencialmente } \\
\hline Região 4 e 2 & Região 4 e 5 & Região 4 e 6 \\
\hline 90,9 & 85,0 & 87,8 \\
\hline
\end{tabular}

De acordo com a Tabela 6, ao tocar o instrumento sequencialmente com as mãos alternadas, observa-se que cada região excitada emite o seu som de modo a não interferir na próxima região a ser excitada.

\section{RESULTADOS}

O resultado final é o mapeamento das frequências emitidas pelo instrumento quando tocado, conforme mostrado na Figura 8.

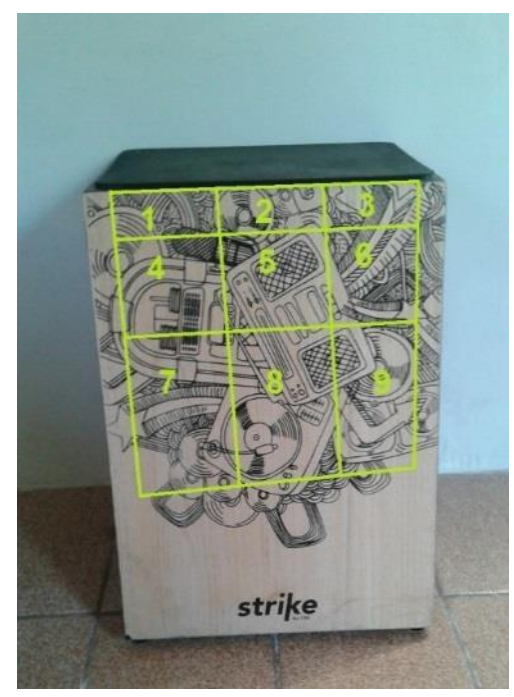

Figura 8. Malha gerada para o mapeamento sonoro do cajón.

Em seguida foram verificados os espectros de frequências das regiões tocadas livremente e com bloqueio nas regiões 3 e 9 , a fim de se descobrir quais frequências possuem maiores amplitudes.

A Figura 9 mostra o espectro de frequências da região 3 tocada livremente. 


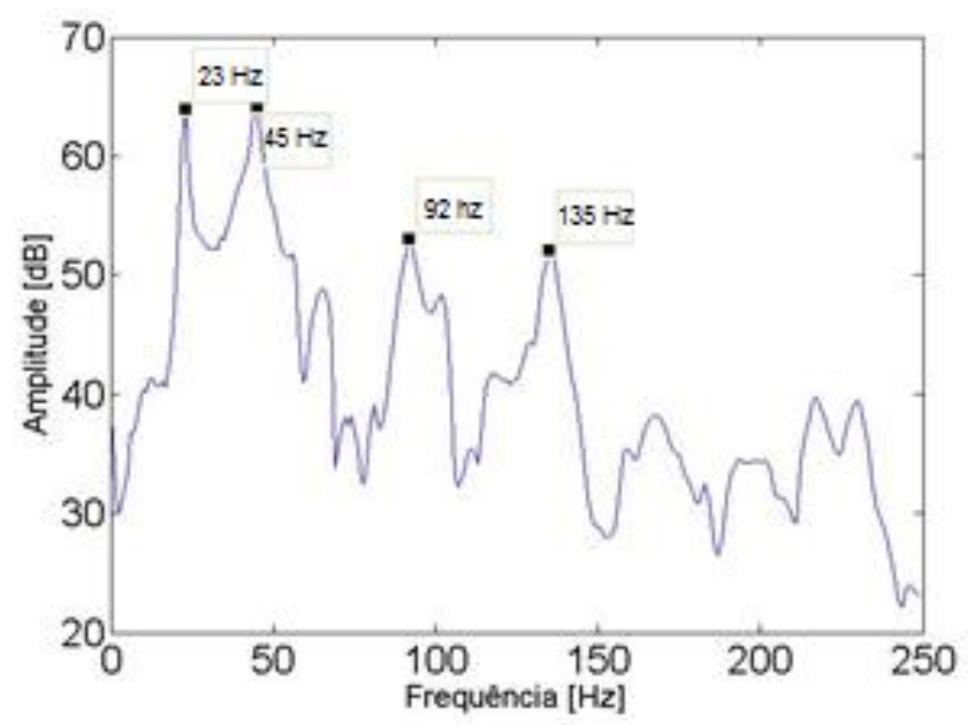

Figura 9. Espectro de frequências da região 3 tocada com o tampo livre.

Como pode ser visualizado na Figura 9, ao tocar a região 3, elevadas amplitudes podem ser notadas em mais de uma frequência. Após a análise de todos os espectros de frequências, foi possível constatar que o instrumento emite elevadas amplitudes em aproximadamente quatro frequências.

Os resultados são apresentados a seguir.

- Valores de frequência com maiores amplitudes para cada região do cajón tocado livremente (valores na faixa de $1 \mathrm{~Hz}$ a $40 \mathrm{~Hz}$ ):

\begin{tabular}{|l|l|l|}
\hline 23 & 22 & 23 \\
\hline 23 & 38 & 23 \\
\hline 39 & 22 & 38 \\
\hline
\end{tabular}

- Valores de frequência com maiores amplitudes para cada região do cajón tocado livremente (valores na faixa de $41 \mathrm{~Hz} \mathrm{a} 51 \mathrm{~Hz}$ ): 


\begin{tabular}{|l|l|l|}
\hline 45 & 45 & 45 \\
\hline 44 & 51 & 45 \\
\hline 51 & 45 & 51 \\
\hline
\end{tabular}

- Valores de frequência com maiores amplitudes para cada região do cajón tocado livremente (valores na faixa de $52 \mathrm{~Hz}$ a $102 \mathrm{~Hz}$ ):

\begin{tabular}{|c|c|c|}
\hline 58 & 67 & 92 \\
\hline 57 & 65 & 64 \\
\hline 93 & 83 & 91 \\
\hline
\end{tabular}

- Valores de frequência com maiores amplitudes para cada região do cajón tocado livremente (valores na faixa de $103 \mathrm{~Hz}$ a $140 \mathrm{~Hz}$ ):

\begin{tabular}{|l|l|l|}
\hline 135 & 134 & 135 \\
\hline 103 & 102 & 103 \\
\hline 135 & 123 & 128 \\
\hline
\end{tabular}

- Valores de frequência com maiores amplitudes para cada região do cajón tocado com bloqueio na região 3 (valores na faixa de $1 \mathrm{~Hz}$ a $40 \mathrm{~Hz}$ ): 


\begin{tabular}{|l|l|l|}
\hline 27 & 25 & $X$ \\
\hline 29 & 25 & - \\
\hline 39 & 38 & - \\
\hline
\end{tabular}

- Valores de frequência com maiores amplitudes para cada região do cajón tocado com bloqueio na região 3 (valores na faixa de $41 \mathrm{~Hz}$ a $51 \mathrm{~Hz}$ ):

\begin{tabular}{|c|c|c|}
\hline 46 & 46 & $X$ \\
\hline 49 & 45 & - \\
\hline 48 & 49 & - \\
\hline
\end{tabular}

- Valores de frequência com maiores amplitudes para cada região do cajón tocado com bloqueio na região 3 (valores na faixa de $52 \mathrm{~Hz}$ a $102 \mathrm{~Hz}$ ):

\begin{tabular}{|c|c|c|}
\hline 102 & 96 & $x$ \\
\hline 65 & 96 & - \\
\hline 93 & 81 & - \\
\hline
\end{tabular}

- Valores de frequência com maiores amplitudes para cada região do cajón tocado com bloqueio na região 3 (valores na faixa de $103 \mathrm{~Hz}$ a $140 \mathrm{~Hz}$ ): 


\begin{tabular}{|c|c|c|}
\hline 136 & 131 & $X$ \\
\hline 109 & 133 & - \\
\hline 133 & 126 & - \\
\hline
\end{tabular}

- Valores de frequência com maiores amplitudes para cada região do cajón tocado com bloqueio na região 9 (valores na faixa de $1 \mathrm{~Hz}$ a $40 \mathrm{~Hz}$ ):

\begin{tabular}{|c|c|c|}
\hline 29 & 29 & 25 \\
\hline 26 & 28 & 25 \\
\hline 24 & 38 & $X$ \\
\hline
\end{tabular}

- Valores de frequência com maiores amplitudes para cada região do cajón tocado com bloqueio na região 9 (valores na faixa de $41 \mathrm{~Hz}$ a $51 \mathrm{~Hz}$ ):

\begin{tabular}{|c|c|c|}
\hline 47 & 48 & 64 \\
\hline 45 & 46 & 45 \\
\hline 46 & 69 & $X$ \\
\hline
\end{tabular}

- Valores de frequência com maiores amplitudes para cada região do cajón tocado com bloqueio na região 9 (valores na faixa de $52 \mathrm{~Hz}$ a $102 \mathrm{~Hz}$ ): 


\begin{tabular}{|c|c|c|}
\hline 80 & 89 & 97 \\
\hline 66 & 85 & 83 \\
\hline 81 & 89 & $X$ \\
\hline
\end{tabular}

- Valores de frequência com maiores amplitudes para cada região do cajón tocado com bloqueio na região 9 (valores na faixa de $103 \mathrm{~Hz}$ a $140 \mathrm{~Hz}$ ):

\begin{tabular}{|c|c|c|}
\hline 129 & 128 & 238 \\
\hline 123 & 125 & 133 \\
\hline 128 & 117 & $X$ \\
\hline
\end{tabular}

\section{DISCUSSÃO}

Durante os testes observou-se que, quando tocado livremente, o cajón emite com grande intensidade as baixas frequências.

De certa forma, este resultado é esperado, pois o cajón é um instrumento de percussão feito para simular de maneira simplificada o bumbo da bateria, e também toda a sua estrutura tende a liberar um som mais grave.

Também foi observado a existência de maiores amplitudes em frequências na faixa de 55 a $140 \mathrm{~Hz}$, principalmente quando se toca a parte superior do instrumento, onde situa-se a esteira metálica, que serve para simular a caixa da bateria.

Quando uma região é tocada, o instrumento emite tanto as frequências situadas entre 20 e $55 \mathrm{~Hz}$, quanto as frequências situadas entre $55 \mathrm{a} 140 \mathrm{~Hz}$, sendo esperado que quanto mais perto da esteira for a batida, menos grave tende a ser o som, e quanto mais perto do centro do tampo, mais grave tende a ser o som. 
Outra constatação importante são as repetições de frequências nas regiões 1, 2 e 3, ambas emitindo sons com maiores amplitudes em $23 \mathrm{~Hz}, 45 \mathrm{~Hz}$ e $135 \mathrm{~Hz}$. Este resultado de certa forma também é esperado, pois o sistema é simétrico.

\section{CONCLUSÓES}

Foram realizados testes para obter o mapa de frequências do cajón acústico. A repetibilidade foi mantida com a utilização de um pêndulo e um bloco grande com revestimento em EVA em sua extremidade para excitar o cajón durante os testes.

Realizaram-se testes de simetria que demonstraram que quando o instrumento é tocado livremente, sem nenhum tipo de bloqueio interferindo na rigidez do tampo, o instrumento é simétrico sonoramente, emitindo sons nas mesmas frequências nos lados esquerdo e direito. Já considerando algum tipo de bloqueio no tampo, o mesmo não ocorre, devido às mudanças nas propriedades do sistema. Estas mudanças também foram avaliadas, pois bloquear em algum ponto do tampo é uma maneira muito usual de se tocar o instrumento. Sendo assim, após realizar os testes considerando as duas regiões mais usualmente bloqueadas, conclui-se que o instrumento muda as frequências emitidas, ou as notas, fazendo então com que o músico ganhe mais possibilidades sonoras ao tocar.

Reparou-se também que o músico por muitas vezes toca duas regiões ao mesmo tempo. Isto é feito para ganhar mais intensidade sonora, e o mesmo foi verificado nos testes. Os espectros somados das regiões excitadas assemelham-se muito com o espectro excitando as mesmas regiões ao mesmo tempo, concluindo-se então que a sobreposição de sinal neste caso ocorre, e que a tentativa de conseguir maior intensidade sonora ao bater duas regiões ao mesmo tempo é válida.

Sendo assim, com os estudos realizados, pode-se dizer que a construção de um cajón eletrônico é plausível, onde devem ser considerados a simetria, a maneira de tocar livremente e com bloqueio em alguma região e a sobreposição de sinais quando tocadas duas regiões ao mesmo tempo, gerando assim sons diferentes que devem ser incorporados ao instrumento elétrico.

\section{REFERÉNCIAS}

CAVALCANTE, L. C. Avaliação de Novas Espécies Madeireiras na Fabricação de

Cajón. Brasília: Universidade de Brasília, 2006. 
CRISTINO, A. L. Breve Histórico E Caderno De Exercícios Para Estudo De Alguns Ritmos Brasileiros No Cajon. 2008. 25 f. TCC (Graduação) - Curso de Educação Artística-habilitação em Música, Universidade Federal de Uberlândia, Uberlândia, 2008.

FSA CAJÓNS. Strike Series - Cajon FSA Strike Series "The Sixties" -

SK4000/SK5000. Disponível em: <http://www.fsacajons.com.br/produto/cajon-fsa-strikeseries-the-sixties-sk4000sk5000>. Acesso em: 19 abr. 2016.

\section{LIRA, S. A. Análise De Correlação: Abordagem Teórica E De Construção Dos}

Coeficientes Com Aplicações. 2004. 209 f. Dissertação (Mestrado) - Curso de Pós graduação em Métodos Numéricos em Engenharia dos Setores de Ciências Exatas e de Tecnologia da Universidade Federal do Paraná, Universidade Federal do Paraná, Curitiba, 2004.

NATIONAL INSTRUMENTS (Org.). O Que É Aquisição De Dados? Disponível em: <http://www.ni.com/data-acquisition/what-is/pt/>. Acesso em: 20 jun. 2016.

\section{SANTOS, C. V. Teclado Eletrônico: Estratégias E Abordagens Criativas Na}

Musicalização De Adultos Em Grupo. 2006. 183 f. Tese (Doutorado) - Curso de Música, Escola de Música, Universidade Federal de Minas Gerais, Belo Horizonte, 2006. Disponível em: <http://www.bibliotecadigital.ufmg.br/dspace/bitstream/handle/1843/ECOA7KGN8P/introdu_o_e_cap_tulos_1_2_3_4_de_13_de_julho.pdf?sequence=1 $>$. Acesso em: 14 abr. 2016. 